\title{
POLYNOMIAL RINGS OVER JACOBSON-HILBERT RINGS
}

\author{
CARL FAITH
}

\begin{abstract}
All rings considered are commutative with unit. A ring $R$ is SISI (in Vamos' terminology) if every subdirectly irreducible factor ring $R / I$ is self-injective. SISI rings include Noetherian rings, Morita rings, and almost maximal valuation rings ([V1]). In [F3] we raised the question of whether a polynomial ring $R[x]$ over a $S I S I$ ring $R$ is again $S I S I$. In this paper we show this is not the case.
\end{abstract}

\section{Introduction}

The counter-example to the above is provided by the theorem below proved in $\oint 4$.

1.0. Theorem. For every field $K$ and local injective module $E$ of $K[x]$ (= the injective hull of a simple $k[x]-$ module), the split-null extension $A=$ $(K[x], E)$ is subdirectly irreducible, and a factor ring of $R[x]$, where $R$ is the split-null extension $(K, N)$ and $N$ is any vector space over $K$ of dimension not less than that of $E$.

By Example $3.4 \mathrm{~B}, R$ is $S I S I$, but $R[x]$ is not. (See $\oint 4$ for the proof.)

This leaves open the question: Is $R[x] S I S I$ for a Vámos, or even Morita, ring $R$ ?. We also settle another question raised in [F3] by showing that not every SISI ring is "Monica". To define this term, we need two other concepts: (1) an ideal $I$ of co-subdirectly irreducible $(=C O S I)$ if $R / I$ is subdirectly irreducible ring; (2) an ideal $I$ of $R[x]$ is monic if it contains a monic polynomial $\neq 0$ (equivalently, $R[x] / I$ is a finitely generated canonical $R$-module.)

In connection with (2), we first remark:

1.1. Theorem. $R$ is a Jacobson-Hilbert ring iff every maximal ideal of $R[x]$ is monic.

The recessity is stated as an exercise in $[K]$.

Now define Monica ring to a ring $R$ such that every COSI ideal of $R[x]$ is monic. As a corollary to Theorem 1.1, we prove: 
1.2. Theorem. A Noetherian ring $R$ is Monica iff $R$ is Jacobson-Hilbert.

Since any Noetherian ring $R$ is Vamosian (see $\oint 3$ ), hence $S I S I$, this shows these rings need not be Monica. (Cf. Theorem 6.1 which asserts that Von Neumann regular rings are Monica.)

We call a ring $R$ an $H$-ring (after Camillo) if every factor ring modulo a $C O S I$ ideal is a local ring. Obviously any $S I S I$ ring is an $H$-ring. Moreover:

1.3. Theorem. A Jacobson-Hilbert ring $R$ is Monica if $R[x]$ is an $H$-ring.

In Theorem $1.0, R$ is trivially an $H$-ring (since $R$ is local) but $R[x]$ is not (since $A$ is not a local ring). See Theorem 2.5 for a proof of Theorem 1.3.

Every Morita ring is 1.c. (See $\$ 3$.) A conjecture of Zelinsky-Mueller-Vámos $(Z M V C)$ is that every 1.c. ring $R$ is Morita. In [F5] we prove that $Z M V C$ is equivalent to the assertion that every 1.c. ring is SISI.

\section{Proofs of Theorems 1.1 and 1.2}

A domain $R$ is a $G$-domain if its quotient field $K=Q_{C}(R)$ has the equivalent properties:

(G-1) $K=R\left[a_{1}, \ldots, a_{n}\right]$, for finitely many elements $a_{1}, \ldots, a_{n}$

(G-2) $K=R[a]$, for some $a \in K$.

An ideal $I$ is a $G-i d e a l$ iff $I$ satisfies the equivalent properties:

(G-A) $R / I$ is a $G$-domain

(G-A) Some maximal ideal $M$ of $R[x\}$ contracts to $I$.

Let rad $A$ denote the Jacobson radical of $A$ for any ring $A$, i.e. the intersection of all maximal ideals of $A$. An ideal $I$ is a $J$-ideal if the e.c.'s hold:

(1) $\operatorname{rad}(R / I)=0$

(2) $I$ is an intersection of maximal ideals.

Let nil $\operatorname{rad} R$ denote the maximal nil ideal of $R$, i.e.

$$
\text { nil } \operatorname{rad} R=\left\{a \in R \mid 3_{n} a^{n}=0\right\}
$$

If $I$ is an ideal, then $\sqrt{I}$ is the classical radical of $I$, namely the ideal such that

$$
\sqrt{I} / I=\operatorname{nil} \operatorname{rad}(R / I) \text {. }
$$

$R$ is a Jacobson-Hibert ring if $R$ satisfies the e.c.'s:

(J-1) Every $G$-ideal is maximal

(J-2) $\forall$ ideals $I, \sqrt{I}$ is a $J$-ideal, i.e.

$$
\sqrt{I} / I=\operatorname{nil} \operatorname{rad} R / I=\operatorname{rad} R / I
$$

( $J$-3) Every (semi) prime ideal is a $J$-ideal

$(J-4)$ Every $G$-ideal is a $J$-ideal

(J-5) For all maximal ideals $M$ of $R[x], M \cap R$ is a maximal ideal of $R$.

See, e.g., $[K]$, or $[G]$ and $[K r]$. (A Jacobson-Hilbert ring is called a Hilbert ring in $[K]$, and Jacobson ring in [Kr], and in Bourbaki.) 
2.1. Proposition. A maximal ideal $M$ of $R[x]$ is monic iff $M$ contracts to a maximal ideal of $R$.

Proof: Let $M_{O}=M \cap R$ be maximal in $R$. Now $M \supseteq M_{O}[x]$, and $M \neq$ $M_{O}[x]$ that is, $M_{O}[X]$ is not maximal in $R[X]$, since

$$
S=R[x] / M_{O}[x] \approx R / M_{O}[x]
$$

a polynomial ring over a field; thus $M / M_{O}[x]$ is a monic ideal of $S$, hence $M$ is monic in $R[x]$.

Conversely, let $M$ be a monic ideal of $R[x]$. The domain $\bar{R}=R / M_{O}$ embeds canonically in the field $A=R[x] / M$. Let

$$
p(x)=x^{n+1}-\alpha_{O} x^{n}-\cdots-\alpha_{n} \in M
$$

be a monic polynomial with $\alpha_{i} \in R$. For $0 \neq \bar{d} \in \bar{R}, p\left(d^{*}\right) \in M$ where $d d^{*} \equiv 1(\bmod M)$, i.e. $\bar{d}^{*}=\bar{d}^{1} ;$ hence

$$
d^{-(n+1)}=\bar{\alpha}_{0} d^{-n}+\cdots+\bar{\alpha}_{n}
$$

so

$$
1=\left(\bar{\alpha}_{0}+\cdots+\bar{\alpha}_{n} \bar{d}^{n}\right) \bar{d}(\bmod M)
$$

that is,

$$
\bar{d}^{-1}=\bar{\alpha}_{0}+\cdots+\bar{\alpha}_{n} \bar{d}^{n} \in \bar{R}
$$

so $\bar{R}$ is a field, hence $M_{O}$ is maximal, as required.

We say that a ring $R$ is a maxmonica ring if all maximal ideals of $R[x]$ are monic.

2.2. Theorem. A ring $R$ is a maxmonica ring iff $R$ is Jacobson-Hilbert.

Proof: By $J-5$ and Proposition 2.1, any Jacobson-Hilbert ring is a maxmonica ring. Conversely, if $R$ is maxmonica, every $G$-ideal of $R$ is maximal by the proposition, i.e. $R$ is Jacobson-Hilbert $(J-1)$.

2.3. Theorem. A Noetherian ring $R$ is Monica iff $R$ is Jacobson-Hilbert.

Proof: One way by the last theorem. Conversely, if $R$ is Jacobson-Hilbert, and $I$ in $R[x]$ is COSI, then $A=R[x] / I$ is $Q F$ (since Noetherian rings are $S I S I$ by [V1]; also see 3.3B below in $\oint 3$ ), hence $A$ is Artinian with nilpotent radical $M / I$. Then $M$ is a maximal ideal $=\sqrt{I}$, so $M$ monic implies $I$ monic. 
2.4. Proposition. If $R$ is a local Jacobson-Hilbert ring with radical $J$, then an ideal $I$ of $R[x]$ is contained in just finitely many maximal ideals (equivalently $I$ is co-semilocal) iff $I$ is monic.

Proof: If $k=R / J$, then $k[x]$ is a principal ideal domain, and hence every nonzero ideal is co-semilocal in fact co-Artinian. Since $k[x]=R[x] / J[x]$, the same is true for any ideal $K$ of $R[x]$ containing $J[x]$ properly. Now every maximal ideal of $R[x]$ containing $I$ also contains $I+J[x]$, which properly contains $J[x]$ if $I$ is monic, so there are only finitely of them.

The converse does not use the local ring hypothesis. Since $R$ JacobsonHilbert implies that $R[x]$ is Jacobson-Hilbert, if $R[x] / I$ is semilocal, then $\sqrt{I} / I$ is the intersection of just finitely many maximal ideals $\left\{M_{i} / I\right\}_{i=1}^{n}$, so $\sqrt{I}=$ $\cap_{i=1}^{n} M_{i}$ contains the product $\Pi_{i=1}^{n} M_{i}$. But each $M_{i}$ is monic by Theorem 1.1, hence $\Pi_{i=1}^{n} M_{i}$ whence $\sqrt{I}$, whence $I$ is monic.

2.5. Theorem. The face.c.'s on $R$ :

(1) $R$ is Jacobson-Hilbert

(2) Every ideal $I$ of $R[x]$ contained in a unique maximal ideal is monic.

(3) Every ideal $I$ of $R[x]$ contained in just fnitely many maximal ideals is monic.

Proof: (1) $\Rightarrow$ (3) by the proof of one part (the converse) of the last theorem, and $(3) \Rightarrow(1)$ by Theorem 1.1. Similarly, for (1) $\Leftrightarrow(2)$.

A corollary of Theorem 2.5 is Theorem 1.3 (See Proposition 5.4.)

\section{Morita, Vamosian, and SISI Rings}

$A$ ring $R$ is Vamosian, or a Vámos ring, provided that the injective hull $E(R / M)$ is linearly compact (1.c.) in the discrete topology for all maximal ideals $M$. See [V1] and [F3] for background, and the basic theorems. We list a few of these:

3.1. Locally Noetherian rings, i.e. $R_{M}$ is Noetherian for $M \in \max R$ (see [V1]). Any polynomial ring $R[x]$ is then locally Noetherian ([F3]). The basic facts harken back to Matlis' classic paper [Ma].

3.2. Morita rings, i.e. both $R$ and the minimal injective cogenerator $E$ over $R$ are 1.c. $R$-modules (Mueller [Mu]). An equivalent formulation:

$$
R=\operatorname{End}_{R} F
$$

canonically, where $F$ is an injective cogenerator of $\bmod -R$ (Morita $[\mathrm{Mo}]$ ). Then there is a Morita duality induced by $\operatorname{Hom}_{R}(, F)$ on the 1.c. $R$-modules. If $R_{M}$ is Noetherian, then $A=\operatorname{End}_{R} E(R / M)$ is 1.c., and $\operatorname{Hom}_{A}(, E(R / M))$ induces a Morita duality on the 1.c. modules ([Ma],[Mo] and [Mu]). 
3.3A. $A$ ring $R$ is right $P F$ provided $R$ is an injective cogenerator as a right $R-$ module, equivalently, $R$ is right self-injective, and has finite essential right socle. Then, by Morita's theorem, there is a Morita duality, induced by $\operatorname{Hom}_{R}(, R)$, when $R$ is a 2 -sided $P F$ ring.

3.3B. The $Q F$ (= quasi-Frobenius rings are the Artinian (or Noetherian) right (or left) self-injective rings. Every $Q F$ ring is right and left Artinian and right and left $P F$; and conversely a left or right Artinian or Noetherian right or left $P F$ ring is $Q F$. (See, e.g. [F2], Chap. $24 \mathrm{ff}$.)

3.4A. Theorem (Vámos). Morita rings are Vamosian, and Vámos rings are SISI, but not conversely.

3.4B. Example (Vámos [V1j) Let $R$ be any local ring with square-zero radical $N$. Then, $R$ is $S I S I$, and f.a.e.:

(1) $R$ is Vámos

(2) $R$ is Morita

(3) $\operatorname{dim}_{R / N} N<\infty$.

Proof: This is essentially in [V1]. If $I$ is $C O S I$ in $R$, then either $I=N$ or $I \subset N$. In the latter case $N / I$ must be simple, so $A / I$ is Artinian of length 2. Now any semilocal Vamosian ring has finite uniform or Goldie dimension [V1], hence $R$ is Vámos (Morita) if (3) holds.

3.4C. By [VI] and [F3], $R$ is locally SISI iff every local endomorphism ring (= End ${ }_{R} E(V)$, where $V$ is a simple $R$-module, and $E(V)$ is its injective huil) is commutative.

\section{Proof of Theorem 1.0}

A subdirect irreducible (injective) module is an (injective) module $E$ with simple essential socle $V$. In case $E$ is injective, then $E=E(V)$ is a local injective module ([F3]).

Proof: Theorem 1.0 is one of those increasingly familiar theorems in which the statement contains the proof (practically).

Any injective module $E$ over a ring $T$ is divisible by all regular elements of $T$, hence for a domain $T=K[x], E$ is divisible. This implies that $(0, E)$ is a waist in $A$ since if $(a, x) \in A$, and $a \neq 0$, then

$$
(a, x)(0, E)=(0, a E)=(0, E) .
$$

Since $E$ is subdirect irreducible, this implies that $A$ is also.

Let $c$ be a cardinal of a generating set for $E$ over $K[x]$, let $F$ be the free $K[x]$-module on $c$ letters, so there is an exact sequence of $K[x]$-modules

$$
0 \rightarrow I \rightarrow F \rightarrow E \rightarrow 0 .
$$


Note that if $N=L^{(c)}$, the direct sum of $c$ copies of $K$, then

$$
F=K[x]^{(c)} \approx K^{(c)}[x]=N[x] .
$$

Now $N$ is a $K$-module and the split-null extension $R=(K, N)$ has the required property, namely, there is a ring epimorphism

$$
R[x] \rightarrow A .
$$

We use without proof the fact that there is a ring isomorphism

$$
h\left\{\begin{array}{l}
R[x] \approx(K[x], N[x]) \\
\sum_{i=0}^{t}\left(\alpha_{i}, n_{i}\right) x^{i} \rightarrow\left[\sum_{i=0}^{t} \alpha_{i} x^{i}, \sum_{i=0}^{t} n_{i} x^{i}\right]
\end{array}\right.
$$

for $\alpha_{i} \in K, n_{i} \in N, i=0, \ldots, t<\infty$.

Then, we use the ring homomorphism

$$
\left\{\begin{array}{l}
(K[x], N[x]) \rightarrow A=(K[x], E) \\
\left(f_{1}(x), f_{2}(x)\right) \longrightarrow\left(f_{1}(x), \overline{f_{2}(x)}\right)
\end{array}\right.
$$

where $f_{1}(x) \in K[x], f_{2}(x) \in N[x]$, and $f_{2}(x)$ maps onto $\overline{f_{2}(x)}$ under the $K[x]-$ module homomorphism $N[x] \rightarrow E$ defined by (3.5.1) and (3.5.2). (Hint: use the fact that $\overline{f(x) g(x)}=\overline{f(x)} g(x)$ for $f(x) \in K[x]$ and $g(x) \in N[x]$, i.e. $N[x] \rightarrow E$ is a $K[x]$-module homomorphism.)

Finally, since $A$ is not a local ring (also not self-injective), then $R[x]$ is neither a $S I S I$, nor $H$ ring.

We proof a partial converse of Theorem 1.0 .

4.1. Theorem. If $I$ is a non-monic COSI ideal of a polynomial ring $R[x]$ over a Jacobson-Hilbert local ring $R$, then $I \subset N[x]$, where

$$
N=\operatorname{rad} R=\operatorname{nil} \operatorname{rad} R \text {. }
$$

Next assume $N^{2}=0$. Then $A=R[x] / I$ is the trivial extension $(K[x], E)$ where

$$
E=\overline{N[x]}=N[x] / I
$$

is divisible, hence injective, whence local injective $K[x]-$ module.

Proof: A Jacobson-Hilbert local ring $R$ has nil Jacobson radical since the nil radical $N$ must be the (intersection of the) unique maximal ideal. Then $\sqrt{I}$ contains $N[x]$, and since

$$
R[x] / N[x] \approx R / N[x]
$$


and $R / N$ is a Monica ring, then $\sqrt{I}=N[x]$, consequently $I \subseteq N\{x]$. But $I \neq N\{x\}$, since $N[x]$ is not COSI.

Now let $N^{2}=0$. We first assume $I \cap R=0$. If $0 \neq \alpha \in N$, then $\alpha \in I$, hence

$$
\overline{\alpha R[x]} \supseteq \mathrm{V}
$$

where $V=\operatorname{soc} A$, and $\overline{f(x)}$ is the image of any $f(x) \in R[x]$, under the canonical map $R[x] \rightarrow A$. Let $V=(v)$, and write

$$
v=\bar{\alpha} \overline{g(x)}
$$

for some $g(x) \in R[x]$. If $V \approx R[x] / M$, where $M$ is maximal in $R[x]$, then $M$ is generated modulo $N[x]$ by a monic polynomial $m(x)$, and hence $\bar{M}=(\bar{m}, \overline{N[x]})$. Since $\overline{N[x]}^{2}=0$, then $\overline{g(x)} \notin N[x]$. Write

$$
g(x)=m^{t} g_{1}+h
$$

where

$$
\left(m, g_{1}\right)=1(\operatorname{modulo} N[x]),
$$

and $h \in N[x], t \geq 0$. Since $N^{2}=0$, then $\alpha h=0$, hence

$$
v=\overline{\alpha g(x)}=\alpha \bar{m}^{t} \overline{g_{1}} \text {. }
$$

Now $\bar{m} v=0$, hence

$$
\overline{\alpha m}^{t+1} \overline{g_{1}}=0 .
$$

But the regular elements of $A$ are those $\overline{f(x)}$ with $f(x) \notin M$, i.e. $\overline{g_{1}}$ is regular, so $\overline{\alpha m}^{t+1}=0$, that is, $\bar{\alpha}$ annihilates a power of $\bar{m}$.

Expressed otherwise,

$$
N \approx \bar{N} \subseteq \cup_{n=1}^{\infty}\left(\bar{m}^{n}\right)^{\perp}
$$

where $\bar{f}^{\perp}$ is the annihilator in $A$ of any $\bar{f} \in A$.

It is easy to see that if $Q \supseteq I$ is such that

$$
\bar{Q}=\cap \bar{m}^{n},
$$

then

$$
\bar{Q}=\overline{m Q}=\bar{m}^{n} \bar{Q} \forall n \geq 0 .
$$

This follows, since if $\bar{q} \in \bar{Q}$, then

$$
\bar{q}=\overline{m a}_{1}=\bar{m}^{2} \bar{a}_{2}=\ldots
$$

for suitable $\bar{a}_{i} \in A$, and then

$$
a_{1}-\bar{m} a_{2} \in \bar{m}^{\perp} .
$$


Since

$$
V=\bar{M}^{\perp}=\bar{m}^{\perp} \cap \overline{N[x]}^{\perp}=\bar{m}^{\perp} \cap \overline{N[x]}=\bar{m}^{\perp},
$$

is contained in every ideal $\neq 0$ of $A$, the $\bar{m}^{\perp} \subseteq(\bar{m})$, so $\bar{a}_{1} \in(\bar{m})$. By induction, every $\bar{a}_{i} \in(\bar{m})$, consequently $\bar{a}_{1} \in\left(\bar{m}^{i}\right) \bigvee_{i}$. This proves that $\bar{a}_{1} \in \bar{Q}$, hence that $\bar{Q} \subseteq \overline{m Q}$, that is, $\bar{Q}=\bar{m} \bar{Q}$, whence $\bar{Q}=\bar{m}^{n} \bar{Q} \forall_{n}$.

Since $I$ is not monic, $m^{n} \notin I$ hence $m^{n}+I \supseteq I$, so $\bar{m}^{n} \supseteq V \forall_{n}$. Thus $\bar{Q} \supseteq V$. Let $\bar{H}_{n}=\left(\bar{m}^{n}\right)^{\perp}$. Then

$$
\bar{H}_{1}=\bar{m}^{\perp}=\bar{m}^{\perp} \cap \overline{N[x]}=\bar{M}^{\perp}=V .
$$

Suppose $\bar{H}_{n} \subseteq Q$, and let $\bar{u} \in \bar{H}_{n+1}$. Then $\bar{m} \bar{u} \in \bar{H}_{n} \subseteq \bar{Q}=\overline{m Q}$, so $\overline{m u}=\overline{m q}$ for some $\bar{q} \in Q$. Then $\bar{h}=\bar{u}-\bar{q} \in \bar{m}^{\perp}=V \subseteq Q$ hence

$$
\bar{u}=\bar{h}+\bar{q} \in Q \text {. }
$$

This proves that

$$
\bar{H}=\cup_{n=1}^{\infty} \bar{H}_{n} \subseteq \bar{Q}
$$

Now, by (1) and (2),

$$
\bar{N} \subseteq \bar{N}[x] \subseteq \bar{H} \subseteq \bar{Q} .
$$

And since $\overline{N[x]}$ is a prime ideal, it contains $\bar{f}^{\perp} \forall \bar{f} \neq 0 \in A$, so

$$
\bar{H}=\overline{N[x]} \subseteq \bar{Q} .
$$

Since $R[x] / N[x]=K[x]$ is a polynomial ring over a field, then

$$
n_{n=1}^{\infty}\left(m^{n}\right) \subseteq N[x]
$$

so

$$
\bar{H}=\bar{Q}=\cap\left(\bar{m}^{n}\right)=\overline{N[x]} .
$$

Now $F=R[x] / M$ is a field, and $\bar{H}_{n+1} / \bar{H}_{n}$ is a vector space over $F$, hence divisible by every $0 \neq t \in F$, and therefore, $\overline{H f}=\bar{H}$ for every $\bar{f} \in A / \bar{M}$. Since

$$
\overline{H m}^{n}=\overline{Q m}^{n}=\bar{Q}=\bar{H},
$$

then $E=\overline{N[x]}$ is divisible by every $0 \neq f(x) \in K[x\}$ (using (4)).

By the known theory of injective modules over a PID (see, e.g. [F1]), then $E$ is injective. Since $E$ is subdirect irreducible, then $E$ is a Iocal injective $K[x]$-module, and evidently $A$ is the trivial extension $(K[x], E)$.

This completes the proof once we remove the condition $I \cap R=0:$ if $I_{O}=$ $I \cap R$, then $I \supseteq I_{O}[x]$, and $A=R[x] / I$ is an epic image of the polynomial ting

$$
R[x] / I_{O}[x] \approx\left(R / I_{O}\right)[x]
$$

that is,

$$
A \approx\left(R / I_{O}\right)[x] /\left(I / I_{O}\right)[x]
$$

Moreover,

$$
\left(I / I_{O}\right) \cap\left(R / I_{O}\right)=0 .
$$

Now $R / I_{O}$ is a local ring if $R$ is, and also has square zero radical if $R$ does. The conclusion of the theorem is therefore valid for any COSI ideal $I$. 
4.2. Theorem. Let $R$ be a semilocal ring with radical $J$. Then $R[x]$ is SISI only if $J / J^{2}$ is finitely generated.

Proof: $\vec{R}=R / J^{2}$ is a semiprimary ring, hence a finite product of radical square-zero local rings, hence assume $\bar{R}$ is a local ring. Since $\bar{R}[x]$ is a factor ring of $R[x]$, then $\bar{R}[x]$ is $S I S I$ by [F3], hence theorem 1.0 implies that $\operatorname{dim}_{\bar{R}} \bar{J}<\infty$, i.e. $J / J^{2}$ is finitely generated.

4.3. Theorem. If $R$ is a perfect ring, then $R[x]$ is $S I S I$ iff $R$ is Artinian.

Proof: By a theorem of Osofsky [0] a perfect ring $R$ is Artinian iff $J / J^{2}$ is finitely generated. By a theorem of Bass, a perfect ring $R$ is semiperfect and has radical $J \neq J^{2}$. The theorem now applies to complete the proof.

A valuation ring $R$ is discrete $V R(=D V R)$ provided that $R$ satisfies the e.c.'s:

$(D V R 1) R$ is a Principal ideal ring $(P I R)$

(DVR2) $R$ is Noetherian

In this case, $\cap_{n \in \omega} J^{n}=0$ by the Krull Intersection theorem.

Remark. For convenience below, we allow the possibility that $J^{n}=0$ for some $n$.

4.4 Theorem. If $R$ is a SISI VR, equivalently, an $A M V R$, and if $J=$ $\operatorname{rad} R \neq J^{2}$, then $R[x]$ is SISI only if $\bar{R}$ is Noetherian, that is, only if $\bar{R}$ is a $D V R$, where $\bar{R}=R / P$, and $P=n_{n \in \omega} J^{n}$.

Proof: If follows from theorem 4.3 that $\vec{R}[x]$ is $S I S I$ iff $R / J^{2}$ is Artinian. Then $\bar{R}$ is a Noetherian $V R$, whence $D V R$, so $\bar{J}=\bar{x} \bar{R}$ for some $x \in R$.

But, then $x \notin J^{2}$, hence $x R \supset J^{2}$, so $J=x R$.

This implies that $\bar{R}$ is Noetherian, whence a $D V R$.

4.5. Example. Let $R=(B, E)$ be the split-null extension of a $D V R B$ and the least injective cogenerator $E$ over $B$. Then $R$ is an $A M V R$, and $\tilde{R}=R / P$ is a $D V R$. Actually, in this case $R$ is $P F$, by Theorem 2 of [F4]. Similarly, in Theorem 4.4, we have the:

4.6. Corollary. Under the assumptions of the theorem, if $P \neq P^{2}$, then either

(1) $P / P^{2} \approx Q_{C}(\bar{R})$, or

(2) $E=P / P^{2}$ is the least injective cogenerator of $\bar{R}$, and $R / P^{2} \approx(\bar{R}, E)$ is $P F$.

Proof: It follows easily from the theorem that $P$ is divisible by $p^{n}$ for every $n$, so $P / P^{2}$ is divisible over $\bar{R}$, hence injective. Since $P / P^{2}$ is uniform, then 
$P / P^{2}$ is indecomposable, and accordingly either torsion-free, or else torsion. Then (1) holds in the former case. IF $E=P / P^{2}$ is torsion, then it is the least injective cogenerator over $\bar{R}$.

Since $\bar{R}$ is Morita, then $\bar{R}=\operatorname{End}_{\bar{R}} E$, so $R \approx(\bar{B}, E)$ is $P F$ by Lemma 1 and Theorem 2 of [F4].

\section{Polynomial rings over Morita rings}

In this section we investigate $R[x]$ for $R$ a Morita ring. By a theorem of Vámos [V1],[V2], if $A$ is a ring extension of $R$, and if $A$ is a 1.c. $R$-module, e.g., if $A$ is finitely generated $R$-module, then $A$ is also Morita. This implies that $A=R[x] / I$ is Morita for any monic ideal $I$ of $R[x]$.

5.1. Theorem. 1. If $R$ is Jacobson-Hilbert, and if a factor ring $R[x] / I$ is 1.c. ring, then $I$ is monic.

2. If $R$ is a 1.c. Jacobson-Hilbert ring, then for any monic ideal $I$, the factor $\operatorname{ring} A=R[x] / L$ is 1.c. as a ring.

Proof: Any 1.c. ring is semilocal (in fact, semiperfect-see [S]). Then, $A$ 1.c. implies that $I$ is monic by Corollary 2.3. In this case $R[x] / I$ is a finitely generated $R$-module, whence 1.c. as an $R$-nodule, whenever $R$ is. (See, e.g., [V1].) The converse is trivial if $A$ is 1.c. as an $R$-module.

5.2. Corollary. If $R$ is Jacobson-Hilbert, then for any ideal $I$ of $R[x]$, $R[x] / I$ is Morita only if $I$ is monic. This holds in particular, when $R[x] / I$ is $P F$ (or $Q F)$.

Proof: A Morita ring is 1.c. and a $P F$ (also $Q F$ ) ring is a Morita ring.

(Part of the next result is Theorem 1.3 of the Introduction.)

5.3. Proposition. For a Jacobson-Hilbert ring $R$, consider the following $s$ conditions:

(1) $R[x]$ is $S I S I$

(2) $R[x]$ is an $H$-ring

(3) Every COSI ideal $I$ of $R[x]$ is contained in just finitely many maximal ideals

(4) $R$ is Monica;

Then (1) $\Leftrightarrow(2) \Leftrightarrow(3) \Rightarrow$ (4) and conversely if $R$ is a Morita ring.

Proof: Any SISI ring is an $H$-ring, so (1) $\Rightarrow(2)$ and (2) $\Rightarrow(3)$ is trivial. Next, $(3) \Rightarrow(4)$ by Theorem 2.5 .

Now assume (4). By the introduction to this section, if $R$ is Morita, then $A=R[x] / I$ is Morita, hence $S I S I$ for any COSI ideal $I$, and therefore selfinjective. Thus $R[x]$ is $S I S I$, so (4) $\Rightarrow(1)$, assuming $R$ is Morita.

Any Monica ring is Jacobson-Hilbert, so we also have: 
5.4. Corollary. If $R$ is a Monica Morita ring, then $R[x\}$ is SISI.

\section{Von Neumann regular rings are Monica}

By a theorem of Kaplansky, a von Neumann regular (VNR) ring $R$ has the (characterizing) property that $R_{M}$ is a feld for each maximal ideal $M$, and hence, as Vámos pointed out in [VI], is Vamosian, whence SISI (see $\oint 3.1$ ). Moreover, $R$ is Jacobson-Hilbert since every prime ideal is maximal.

\subsection{Theorem. Any $V N R$ ring $R$ is Monica.}

Proof: $R$ is Jacobson-Hilbert, and by 3.1 the ring $R[X]$ is $S I S I$, hence $R$ is Monica by Prop. 5.3.

A ring $R$ is Prüfer (also called Arithmetical) iff $R_{M}$ is a $V R$ for all maximal ideals $M$. Any semihereditary ring is a Prüfer ring, since then $R_{M}$ is a valuation domain $(V D)$ for every maximal ideal.

6.3. Corollary. A Prüfer ring $R$ is SISI iff $R_{M}$ is an AMVR for all maximal ideals $M$.

Proof: By [VI], $R$ is SISI iff $R_{M}$ is SISI $\forall M$. By [F3], any SISI VR is an $A M V R$, so the corollary follows.

6.2. Corollary. If $R$ is a von Neumann regular (VNR) ring, then $R[x]$ is Prüfer and SISI, hence $R[x]_{M}$ is an AMVD for all maximal ideals $M$.

Proof: Over a $V N R$ ring $R$, the polynomial ring $R[x]$ is semihereditary hence Prüfer. Since $R$ is locally Noetherian (in fact locally a field) then so is $R[x]$, so $R[x]$ is $S I S I$. (See $\oint 3.1$ ). Since $R[x]_{M}$ is Noetherian, it is a $D V D$. I have $D_{r}$. P. Pillay to thank for noting this.

\section{Open Problems}

In this paper, we have shown that a polynomial ring over a SISI JacobsonHibert local ring need not be $S I S I$, in fact need not be an $H$-ring. Does the corresponding hold for Vámos or Morita rings? Also similar questions may be asked for a 1.c. $R$, i.e. when is $R[x] / I$ also a 1.c. ring, other than when $I$ is monic?

Characterize $R$ such that all $C O S I$ (or maximal) ideals of $R[x]$ are faithful. These include Monica (maxmonica) rings. 


\section{Note}

Hilbert rings are so-called because of their connections with the Hilbert Nullstellensatz (see [K] for a lucid exposition of Goldman's [G] and Krull's [Kr] results.) Jacobson rings are named by $[\mathrm{Kr}]$ because of their characterizations via the condition that the nilradical equals the Jacobson radical in a any factor ring.

Some of these same ideas have been extended to polynomial rings over von Neumann regular rings by Gentle [Ge]. (Cf. Theorem 6.1 which implics that $V N R$ 's are Monica rings.)

\section{References}

[C]. CAMILLO, V.P., Homological independence of injective hulls of simple modules over commutative rings, Comm. Alg. 6(14) (1978), 145969.

[F1].FAITH, C., "Algebra I: Rings, Modules, and Categories," Springer-Verlag, Berlin, Heidelberg, New York, 1973. corrected reprint, 1981

[F2].FAITH, C., "Algebra II: Ring Theory," Springer-Verlag, 1976.

[F3].FAITH, C., Linearly compact modules and a theorem of Vámos, Publ. Sec. Mat. Univ. Autonoma Barcelona 30 (1986), 127-148.

[F4].FaITH, C., Self-injective rings, Proc. Amer, Math. Soc. 77 (1979), 157-164.

[F5].FAITH, C., A note on Morita and Hnearly compact rings. to appear

[G]. Goldman, O., Hilbert rings and the Hilbert Nullstellensatz, Math. Z. 54 (195I), 136-140.

[Ge].GentLe, R., Comment on the Nullstellensatz for regular rings, Can. Bull. Math. 30 (1987), 124-128.

[K]. Kaplansky, I., Commutative Rings, Univ. of Chicago Press (1974).

[Kr].KRULL, W., Jacobonsche Rings, Hilbertscher Nullstellensatz, Dimensiontheorie, Math. Z. 54 (1951), 354-387.

[Ma] MATLIS, E., Injective modules over Noetherian rings, Pac. J. Math 83 (1958), 207-19.

[Mu]Mueller, B., Linear compactness and Morita duality, J. Algebra 16 (1970), 60-66.

[Mo]MORITA, K., Duality for modules and its applications to the theory of rings with minimum condition, Sci. Reports 6 (1959), 83-142, Tokyo Kyoiku Daigaku.

[V1].VÁmos, P., Classical rings, J. Algebra 34 (1959), 114-129.

[V2].VÁMOS, P., Rings with duality, London Math. Soc. 35 (1977), 275-289.

[V3].VÁMOS, P., The decomposition of finitely generated modules and fractional self-injective rings, J. London Math. Soc. (2) 16 (1977), 209-220. 
[S]. SANDOMIERSKI, F.L., Linearly compact modules and local Morita duality, in Ring Theory, Academic Press (1972), New York.

[Z]. ZELINSKY D., Linearly compact modules and rings, Amer. J. Math. (1953), 79-90.

\author{
Rutgers, The State University \\ New Brunswick \\ NJ 08903 U.S.A. \\ Institut d'Estudis Catalans \\ Centre de Recerca Matemàtica \\ Bellaterra, Apartat 50 \\ 08193 SPAIN
}

Rebut el 4 de maig de 1988 